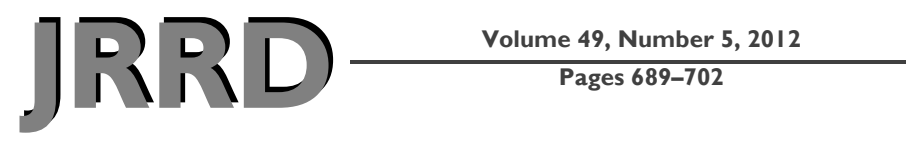

\title{
Review of group treatment for PTSD
}

\author{
Denise M. Sloan, PhD; ${ }^{\mathbf{*}^{*}}$ Michelle J. Bovin, PhD; ${ }^{\mathbf{1}}$ Paula P. Schnurr, $\mathbf{P h D}^{\mathbf{2}}$ \\ ${ }^{1}$ Behavioral Science Division, National Center for PTSD, Boston, MA; Department of Veterans Affairs Boston Health- \\ care System, Boston, MA; and Boston University School of Medicine, Boston, MA; ${ }^{2}$ Executive Division, National Cen- \\ ter for PTSD, White River Junction, VT; and Geisel School of Medicine, Dartmouth College, Hanover, NH
}

\begin{abstract}
The purpose of this article is to provide a brief review of group treatment for posttraumatic stress disorder (PTSD). This review includes a description of group-based treatments for PTSD and the available data on the efficacy of group treatment for PTSD. The literature review indicates that group treatment for PTSD is efficacious compared with no treatment. However, specific types of group treatment are not efficacious when compared with a nonspecific group treatment, such as psychoeducation or supportive counseling. Recommendations for practice and research are made in light of the available literature.
\end{abstract}

Key words: cognitive behavioral therapy, cognitive processing therapy, comparative effectiveness, Department of Veterans Affairs, effect size, group treatment, posttraumatic stress disorder, psychotherapy, review, trauma.

\section{INTRODUCTION}

All types of evidence-based treatments for posttraumatic stress disorder (PTSD), such as cognitive therapy, exposure therapy, and Eye Movement Desensitization and Reprocessing therapy [1], are delivered in individual format [2-3]. Group therapy is not currently recognized as a first-line treatment by the Department of Veterans Affairs (VA) and Department of Defense (DOD) VA/DOD Clinical Practice Guideline for Management of PostTraumatic Stress [4] in the United States or by other PTSD treatment guidelines from around the world [5]. Despite this lack of formal endorsement, the group treatment format is frequently used in healthcare settings [6], including the VA [3,7], where the prevalence of PTSD is relatively high. The purpose of this article is to review the literature on group treatment for PTSD. We

Abbreviations: $\mathrm{AD}=$ adequate dose, $\mathrm{AMR}=$ applied muscle relaxation, CAPS = Clinician-Administered Posttraumatic Stress Disorder Scale, CBT = cognitive behavioral therapy, Comp = completer analysis, $\mathrm{CPT}=$ cognitive processing therapy, CSA = child sexual abuse, DBT = dialectical behavioral therapy, $d f=$ degree of freedom, DOD = Department of Defense, DTS = Davidson Trauma Scale, EMDR = Eye Movement Desensitization and Reprocessing, HIV = human immunodeficiency virus, ICC = intraclass correlation coefficient, IES = Impact of Events Scale, ITT = intention-to-treat, LOCF = lastendpoint-carried-forward, MA = minimal attendance, $\mathrm{MCC}=$ minimal contact condition, MVA = motor vehicle accident, PCL-M = Posttraumatic Stress Disorder Checklist-Military version, PCL-S = Posttraumatic Stress Disorder ChecklistSpecific version, PSS-SR = Posttraumatic Stress Symptom Scale-Self-report, psychoed $=$ psychoeducation, PTSD $=$ posttraumatic stress disorder, RCT = randomized controlled trial, TAU = treatment as usual, TSI-DA = Trauma Symptom Inventory-Defensive Avoidance, TSI-IE = Trauma Symptom Inventory-Intrusive Experiences, VA = Department of Veterans Affairs, WL = wait list.

*Address all correspondence to Denise M. Sloan, PhD; National Center for PTSD (116B-2), VA Boston Healthcare System, 150 S Huntington Ave, Boston, MA 02130.

Email: Denise.Sloan@va.gov

http://dx.doi.org/10.1682/JRRD.2011.07.0123 
first describe the types of group treatments for PTSD that are commonly used in practice. We then describe the recommendations for group treatment from the clinical practice guidelines [4]. Next, we provide a brief review of the literature on the efficacy of group treatment for PTSD and conclude with recommendations for group treatment for PTSD.

\section{METHODS}

We conducted a comprehensive literature review by searching several databases: PsycINFO, Social Sciences Citation Index (SSCI), MedLine, and Published International Literature on Traumatic Stress (PILOTS). We entered various permutations of the words "group," "treatment," "intervention," "therapy," and "psychotherapy" as search criteria, simultaneously with various permutations of the following trauma-related words: "trauma," "posttraumatic stress disorder," "PTSD," "childhood sexual abuse," "rape,” "sexual assault," "physical assault/abuse," "combat," "veteran,” "motor vehicle accident," "domestic violence/abuse," "violence," "natural disaster," "hurricane," "tornado," "earthquake," and "fire." In the PsycINFO database, the query searched the presence of search terms in abstracts and English-language journal articles. In the PILOTS database, results were filtered to present only Englishlanguage journal articles. In the SSCI database, results were filtered to present only English-language articles in the following subject areas: psychiatry, health care sciences and services, clinical psychology, multidisciplinary psychology, substance abuse, psychology, interdisciplinary social sciences, applied psychology, social work, and behavioral sciences. When we identified appropriate articles, we examined the reference sections of those articles for additional relevant articles. We also examined book chapters on group treatments for PTSD but identified no additional articles.

\section{APPROACHES TO GROUP TREATMENT FOR PTSD}

\section{Why Group Treatment?}

Group treatments for PTSD are assumed to involve a number of mechanisms that offer benefit beyond those provided by the individual therapy format. First, patients with PTSD are often socially isolated and have difficulty trusting others [6]. Group treatment provides a safe environment for patients with PTSD to become more socially connected with others and offers the opportunity to build trust. Indeed, Yalom regards the social component (e.g., cohesion, interpersonal learning) of group treatment as a central mechanism through which change occurs [8]. Second, patients with PTSD frequently feel that their PTSD symptoms and trauma experiences are unique to them and that others would not be able to understand the symptoms they are experiencing [6]. Group treatment offers the opportunity to normalize PTSD symptoms by engaging with other individuals who have experienced similar trauma symptoms. Group members may also be able to challenge each other in ways a group leader cannot because of perceived shared status as trauma survivors. Yalom refers to this process as universality [8]. Third, outpatient treatment settings are often understaffed and unable to provide individual treatment to each patient presenting for services. Group treatment can maximize limited staff resources.

\section{Types of Group Treatment Techniques and Strategies}

Approaches to group treatment fall into one of three broad categories: psychodynamic and interpersonal groups, cognitive behavioral therapy (CBT) groups, and supportive groups. The key component of psychodynamic and interpersonal groups is the focus on increasing awareness about internal conflicts and defense mechanisms. Psychodynamic groups might focus on gaining insight about how the trauma influenced the patient's sense of self, affective experience, and interpersonal functioning [9]. Unlike in CBT groups, in psychodynamic groups, trauma material typically arises in an unstructured manner and the pace of discussion of trauma memories is often much slower than that of CBT groups. Interpersonal group treatment stems from the interpersonal psychoanalytic work of Harry Stack Sullivan but also incorporates a CBT approach in terms of its timelimited structure and the use of homework [10]. Interpersonal group treatment focuses on identifying types and patterns of relationship difficulties connected to the traumatic event that are experienced by group members. Psychodynamic and interpersonal groups are typically closed groups; group members all start the group at the same time and continue until group completion. New group members are not permitted to join once the group has started.

CBT groups are also typically conducted in a closed format. These groups include skills training and traumafocused techniques such as exposure and cognitive 
restructuring. Skills-based groups target specific problem areas that are common for patients with PTSD, such as anger [11] and stress [12]. Groups with an exposure component may include imaginal exposure to the trauma memory, through writing [13-14] or speech within the group treatment setting and homework, such as listening to session tapes [15-16]. Groups that include an exposure treatment component can also include in vivo exposures to feared stimuli associated with the traumatic event $[13,15]$. For these exercises, a hierarchy of feared stimuli is developed within the group setting and, most often, patients complete in vivo exposures as assignments outside of the group sessions. Patients then discuss challenges that arose when conducting in vivo exposures and problem-solve as a group how to best address these challenges. Some CBT groups primarily focus on cognitive therapy. For instance, cognitive therapy is a core component of cognitive processing therapy (CPT), which was first described as a group treatment approach [17] but later developed into an individual therapy approach [18], as well as a group and individual combined treatment [19].

CBT groups may include a combination of techniques. For example, groups with an exposure component also commonly include a component of cognitive therapy (i.e., cognitive restructuring) in which common posttraumatic cognitions (e.g., trust, safety, control) are reviewed and the accuracy of these cognitions are challenged. CBT groups also may include skills training with both exposure and cognitive restructuring $[13,15]$. CBT groups typically involve 12 to 16 sessions.

In supportive groups, participants discuss their ongoing stressors and other group members respond by providing feedback, assistance with problem-solving, and emotional support. The core component of supportive groups is the enhancement of interpersonal connections through the giving and receiving of emotional support and feedback. Common experiences among group members are emphasized, involvement from each group member is actively encouraged, and members are praised for displaying adaptive behaviors. These groups focus on the present rather than on past events. Supportive groups are led by therapists, but these groups can also be led by peers [20]. Supportive groups are most often open groups; group members can join or drop out of the group at any time. Usually supportive groups run continuously, that is, there is no specific time frame for the beginning and end of the group. Supportive groups are useful for patients who are unwilling or unable to engage in traumafocused or skills-based groups.

In addition to these approaches, some treatments combine individual and group treatment. Chard evaluated a combination of individual and group CPT [19]. In addition, Turner et al. [21] and Beidel et al. [22] have developed a multiple component behavioral treatment for PTSD called trauma management therapy. Trauma management therapy begins with individual treatment focused on imaginal and in vivo exposure techniques. After completing the individual treatment component, patients then enter group treatment that focuses on improving both general social skills and anger management skills.

One additional group treatment approach is psychoeducation, in which general information is provided regarding common symptoms of PTSD and how PTSD symptoms are maintained. Psychoeducational groups might also include information on the available treatments for PTSD. Psychoeducational groups are ideal as introductory groups for patients that are first entering a clinic. These groups are structured and require only a handful of sessions (e.g., 4 sessions).

The number of group members to include within a specific group is an important consideration. The literature presents some debate about the specific number of participants to include in a PTSD therapy group. For CPT groups, Resick and Schnicke recommend including four to nine participants [23]. The majority of the group treatment studies we reviewed are consistent with this recommendation; they tended to include six to eight participants per group [9,16,24]. However, there are some notable exceptions with somewhat smaller (e.g., 2 members [25]) and larger (e.g., 10-11 members [11,15]) groups represented in the literature. The type of group that is being conducted should be the primary factor in deciding the number of group members to allow. Although there is no empirical evidence to guide the number of group members to be included, there are general considerations that should be taken into account [8]. Supportive groups can generally accommodate a larger number of group members because these groups are relatively unstructured. Generally, CBT groups have fewer members in order to facilitate learning specific skills to manage their PTSD. Thus, therapists might want to limit the number of group members to maximize learning within the group. However, too few group members can also be problematic if members are absent from particular 
sessions and fall behind the rest of the group or drop out of the treatment, thus reducing the nonspecific benefits associated with the group format for the remaining members. Ideally, groups should be led by two therapists in order to best manage the group and balance presentation of material. However, one therapist can usually manage a supportive group.

\section{VA/DOD Clinical Practice Guidelines}

As can be seen from the earlier description, there are a wide variety of group treatments for PTSD. Treatment guidelines can be useful in providing direction regarding which type of groups to offer for PTSD patients. However, the recommendations from the VA/DOD clinical practice guidelines [4] provide limited guidance in selecting group treatment for PTSD.

Based on the current state of the literature, the VA/ DOD clinical practice guidelines recommend that clinicians should "consider" using group treatment for PTSD. That is, there is no recommendation for or against group treatment, because fair evidence was found that group treatment "can improve health outcomes but . . . the balance of benefits and harms is too close to justify a general recommendation" [4]. The clinical practice guidelines state that the comparability of group and individual treatment is unknown because there has been no research examining group treatment relative to individual treatment. This knowledge would greatly inform clinical decisions about the appropriate treatment for a given patient.

The VA/DOD clinical practice guidelines also state that the research literature on group treatment is significantly behind the literature on individual treatment for PTSD. Our review of the literature is consistent with this conclusion. Thus, we focus our review on the available randomized controlled trials (RCTs) and highlight methodological considerations that may affect the interpretability of the existing research.

\section{RESULTS}

There have been several reviews of the research literature on group treatment for PTSD [6,26-27]. These reviews reveal that the majority of trials conducted have consisted of an uncontrolled design; PTSD symptom severity at pretreatment is compared with symptom severity at posttreatment for a single treatment group. Because uncontrolled designs do not control for the pos- sibility that any reduction in symptom severity at posttreatment is caused by a confounding variable, such as the natural course of symptoms or repeated testing, findings from these studies do not provide conclusive evidence about a treatment's benefits. Thus, these studies offer little guidance to clinicians in deciding whether or not to use the group treatment that was studied. Nonetheless, uncontrolled studies can provide important information on the safety of the treatment (e.g., do symptoms increase during course of treatment?), tolerability (e.g., what percentage of participants drop out of the treatment?), and treatment credibility and satisfaction (e.g., do participants believe the treatment makes sense? Do participants like the treatment and believe the treatment was beneficial?).

\section{Review of Meta-Analytic Findings}

Sloan et al. recently conducted a meta-analysis of the 17 RCTs of group treatment for PTSD (Tables 1-2) [27]. The meta-analysis findings revealed a small betweengroup effect size: Cohen's $d=0.24$. Although this effect size is statistically significant, between-group effect sizes from studies of individual treatment for PTSD are typically greater than 1.0 [37]. The studies reviewed were heterogeneous in terms of target therapy, comparison condition, PTSD outcome measure, and trauma sample. Of the 17 RCTs, 6 did not require a PTSD diagnosis for inclusion in the study and 2 examined a treatment that targeted symptoms other than PTSD [28-29] or focused on comorbid diagnoses such as human immunodeficiency virus diagnosis and substance abuse [25,35-36]. We discuss these 17 studies next, with a focus on the methodological considerations that affect the interpretation of the findings.

The typical considerations that apply to individual psychotherapy research also apply to research on group psychotherapy. The most definitive studies are those that use a comparison group, random assignment, careful attention to therapy delivery, reliable and valid measures, blinded assessment procedures, intention-to-treat (ITT) analysis, and appropriate methods for handling missing data $[3,38]$. Three methodological issues are particularly relevant when evaluating the evidence on group psychotherapy. The first issue is the nature of the comparison group, which affects the scientific inferences that can be drawn from a study, as well as both effect size and statistical power. There is no comparable psychotherapeutic analog to a pill placebo; even treatments such as supportive 
Table 1.

Summary of study characteristics of randomized clinical trials of group treatment for posttraumatic stress disorder (PTSD).

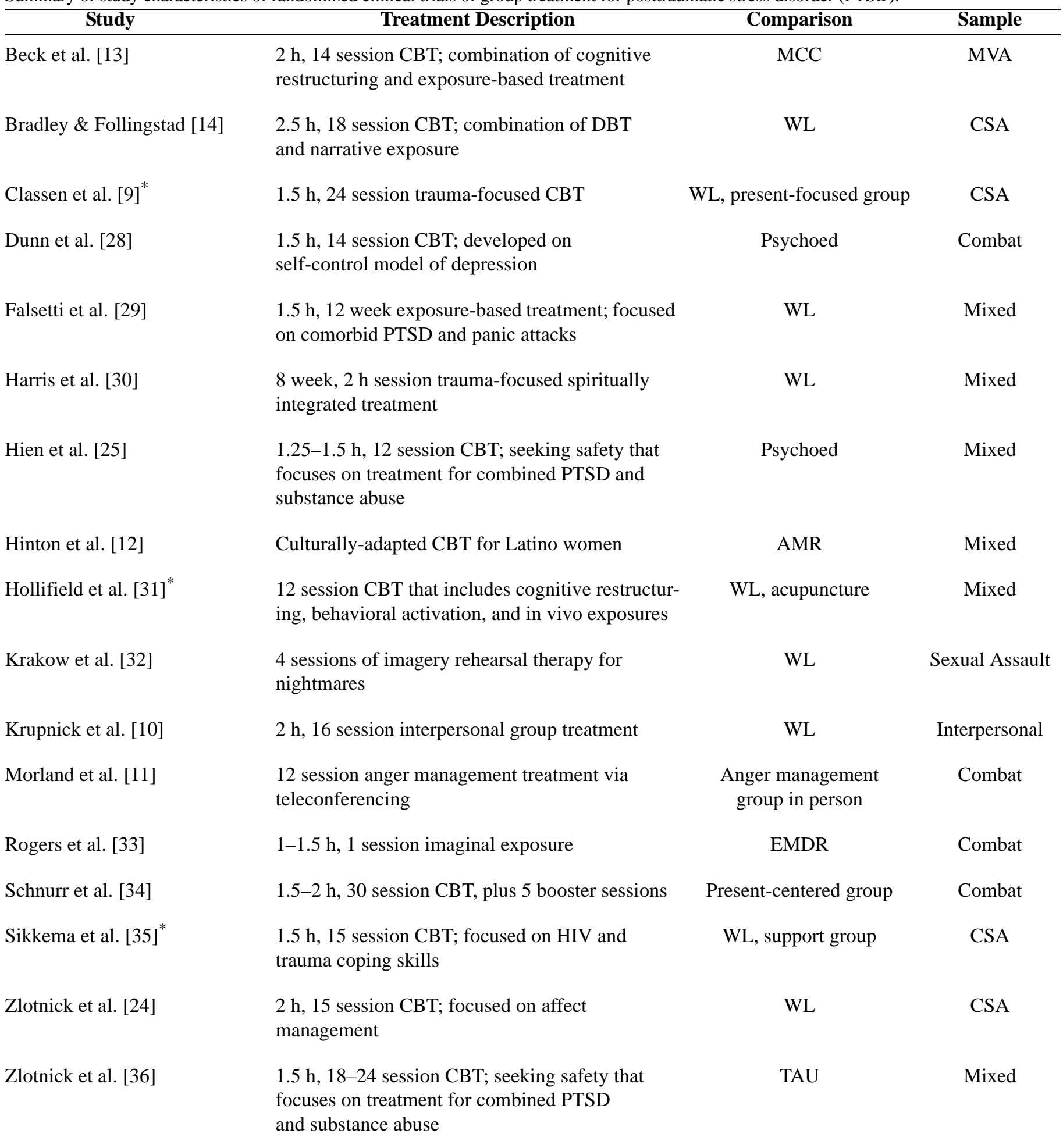

*Included more than 2 conditions.

AMR = applied muscle relaxation, CBT = cognitive behavioral therapy, CSA = childhood sexual abuse, DBT $=$ dialectical behavioral therapy, EMDR $=$ Eye Movement Desensitization and Reprocessing, HIV = human immunodeficiency virus, MCC = minimal contact condition, $\mathrm{MVA}=$ motor vehicle accident, psychoed $=$ psychoeducation, $\mathrm{PTSD}=$ posttraumatic stress disorder, TAU = treatment as usual, $\mathrm{WL}=$ wait list. 
Table 2.

Sample sizes, analysis approach, dropout rates, and outcome measures for each randomized clinical trial of group treatment for posttraumatic stress disorder.

\begin{tabular}{|c|c|c|c|c|c|c|}
\hline \multirow[t]{2}{*}{ Study } & \multirow[t]{2}{*}{ Treatment (n) } & \multirow[t]{2}{*}{ Comparison (n) } & \multirow[t]{2}{*}{ Analysis } & \multirow{2}{*}{$\begin{array}{l}\text { Outcome } \\
\text { Measure }\end{array}$} & \multicolumn{2}{|c|}{$\begin{array}{c}\text { Treatment Dropout Rate } \\
(\%)\end{array}$} \\
\hline & & & & & Treatment & Comparison \\
\hline Beck et al. [13] & 26 & 18 & Comp & CAPS & 27 & 11 \\
\hline Bradley \& Follingstad [14] & 24 & 25 & Comp & TSI-IE, TSI-DA & 46 & 28 \\
\hline Classen et al. [9] ${ }^{*}$ & 55 & $55 / 56$ & AD, ITT, LOCF & PCL-S & 29 & $0 / 14$ \\
\hline Dunn et al. [28] & 55 & 56 & Comp, ITT & CAPS & 38 & 21 \\
\hline Falsetti et al. [29] & $29(22)$ & 31 & $\begin{array}{l}\text { Comp, ITT, } \\
\text { LOCF }\end{array}$ & CAPS & $52(36)$ & 26 \\
\hline Harris et al. [30] & 26 & 28 & Comp & PCL & 6 & 0 \\
\hline Hien et al. [25] & 176 & 177 & MA, ITT & CAPS & $41^{\dagger}$ & $46^{\dagger}$ \\
\hline Hinton et al. [12] & 12 & 12 & ITT & PCL & 0 & 0 \\
\hline Hollifield et al. [31] & 28 & $27 / 29$ & $\begin{array}{l}\text { Comp, ITT, } \\
\text { LOCF }\end{array}$ & PSS-SR & 25 & $22 / 34$ \\
\hline Krakow et al. [32] & 87 & 82 & Comp & PSS-SR & 51 & 41 \\
\hline Krupnick et al. [10] & 32 & 16 & ITT & CAPS & $47^{\dagger}$ & 56 \\
\hline Morland et al. [11] & 61 & 64 & $\mathrm{AD}, \mathrm{ITT}$ & PCL-M & 10 & 11 \\
\hline Rogers et al. [33] & 6 & 6 & ITT & IES & 0 & 0 \\
\hline Schnurr et al. [34] & 180 & 180 & AD, ITT & CAPS & 34 & 25 \\
\hline Sikkema et al. [35] & 96 & $56 / 101$ & ITT & IES & $15^{\dagger}$ & $17 / 15^{\dagger}$ \\
\hline Zlotnick et al. [24] & 16 & 17 & Comp & DTS & 29 & 25 \\
\hline Zlotnick et al. [36] & 27 & 22 & Comp & CAPS & 22 & 0 \\
\hline
\end{tabular}

Note: AD is defined as attending at least $75 \%$ of sessions; MA is defined as attending at least $50 \%$ of sessions.

*Included more than 2 conditions.

${ }^{\dagger}$ Treatment dropout not reported. For Hien et al. and Krupnick et al., dropout rate represents percentage of participants who attended fewer than 50\% of sessions, and for Sikkema et al., dropout rate represents percentage of participants who did not receive any treatment or who were lost to follow-up (i.e., wait list).

$\mathrm{AD}=$ adequate dose, CAPS $=$ Clinician-Administered Posttraumatic Stress Disorder Scale, Comp = completer analysis, DTS $=$ Davidson Trauma Scale, IES = Impact of Events Scale, ITT = intention-to-treat, LOCF = last-observation-carried-forward, MA = minimal attendance, PCL-M = Posttraumatic Stress Disorder Checklist-Military version, PCL-S = Posttraumatic Stress Disorder Checklist-Specific version, PSS-SR = Posttraumatic Stress Symptom Scale-Self-report, TSI-DA = Trauma Symptom Inventory-Defensive Avoidance, TSI-IE = Trauma Symptom Inventory-Intrusive Experiences.

counseling, often used as a comparison group, include nonspecific but essential elements of psychotherapy. Instead, psychotherapy researchers must choose among different comparison conditions depending on the question they want to answer. The second issue is the clustering of observations created by the group format, which has important statistical implications. The third issue, which is important for understanding the results of all clinical trials, is the handling of missing data.

\section{Comparison Groups}

Often the initial stage of research on a therapy uses a wait list or minimal attention comparison group in which participants do not receive study treatment, although stable medication, peer support, and self-help techniques may be allowed. If participants are randomized to treatment, wait list designs control for most potential threats to internal validity and thus permit inferences about whether treatment is responsible for any changes observed. Of the 17 studies listed in Tables 1 and 2, 7 used a wait list comparison group. A limitation of wait list designs is that they offer no information about the mechanism of action. For example, Krupnick et al. found that participants who received group interpersonal therapy had better outcomes relative to participants who were on a wait list [10]. This difference in outcome indicates that the treatment was responsible for the improvement, but not why. Wait list designs do not rule out the possibility that any kind of treatment, or even simply receiving attention from a therapist, would be just as effective or better than the treatment under investigation.

Nonspecific comparison designs that control for the effects of receiving treatment are necessary to determine whether a particular treatment has any unique benefit. Of the 17 studies listed in Tables 1 and 2, 8 used a nonspecific comparison design. Typical nonspecific treatments in these 
studies included relaxation [12], psychoeducation [25,28], present-centered therapy $[9,16]$, and treatment as usual [36]. All but one of these studies [12] found no significant between-group difference in PTSD symptom severity following treatment. However, most of these studies did find that PTSD symptom severity significantly decreased following treatment for both the therapeutic group and the nonspecific comparison condition $[9,16,25,36]$.

Nonspecific comparison groups in combination with a wait list comparison group are helpful for interpreting the results of studies in which the treatments being compared do not differ from one another. The two studies that used present-centered group therapy as a nonspecific comparison treatment for evaluating trauma-focused group CBT $[9,16]$ illustrate this point. Both studies found statistically significant pre-post improvement in each treatment group, and neither study found a difference between trauma-focused CBT and present-centered therapy. Only Classen et al., who found that both treatment groups differed from a wait list group, could definitively attribute the improvement to treatment and not to other factors, such as the natural course of symptoms, repeated testing, or regression to the mean [9]. Of course, interpreting the lack of difference between the two treatments must be tempered by the logical problem of accepting the null hypothesis and the threat of type II error (although Schnurr et al.'s study had high statistical power to detect very small effects [16]). However, findings from both studies suggest that cognitive-behavioral trauma-focused group therapy (at least the types used by these authors) has no effect on PTSD symptoms beyond the effects that result from present-centered treatment.

Although nonspecific comparison designs can provide some information about a treatment's mechanism of action, this question is best addressed by systematically manipulating elements that are thought to be the treatment's active ingredients. No studies of group therapy have used these kinds of designs, even though they could be helpful in determining whether, for example, all of the elements of a particular group treatment are needed.

Comparative effectiveness designs that contrast two or more active treatments comprise the fourth class of psychotherapy research designs. A recent example is the study by Morland et al. comparing two modalities of delivering anger management group therapy: in-person and by videoconference (i.e., the therapist was in a remote location from the therapy group) [11]. These designs may be especially relevant for understanding group therapy because a key question is how group therapy compares with individual therapy. To date, there are no published studies of group therapy compared with individual therapy; however, such studies are currently underway. One type of comparative effectiveness design is the equivalence or noninferiority design. In this design, the null hypothesis is that the treatments differ and the alternative is that the treatments do not differ or that one treatment is not less effective than the other by a prespecified amount [39]. Because they used a noninferiority design, Morland et al. were able to conclude that videoconferencing was not less effective than in-person therapy [11].

The type of design affects more than the interpretation of a study's findings; it also affects the size of the treatment effect [38]. Wait list designs typically yield larger effects than nonspecific designs, which in turn yield larger effects than designs in which active treatments are compared. In Sloan et al.'s meta-analysis, studies that included a wait list comparison condition had a significantly greater between-group effect size $(d=0.54)$ than studies that included an active comparison condition (usually a nonspecific treatment such as present-centered therapy; $d=0.09$ ) [27]. The between-group effect size for studies using an active treatment comparison condition was very small and nonsignificant, indicating that the targeted group treatments did not differ from group treatments intended to control for the nonspecific benefits of group therapy.

\section{Group Clustering}

The essential idea behind all types of group therapy is that members will positively influence one another. The potential advantages of group treatment that come from this mutual influence also create methodological challenges because members' outcomes may be correlated [34,40-41]. The within-group correlation is represented by the intraclass correlation coefficient (ICC). Schnurr et al. reported an ICC of 0.04 for PTSD severity in their RCT [34], and Creamer et al. reported an ICC of 0.13 in an uncontrolled study [42]; an ICC greater than 0 violates assumptions of independence in standard analytic models. Failing to account for the group structure also causes the degrees of freedom (dfs) to be incorrectly estimated. The dfs should be based on the number of groups, not the number of participants. 
The consequence of such failure is that treatment effects will be overstated. Baldwin et al. [40] demonstrated this dramatically with an analysis of findings from 33 studies of group therapy on the list of empirically supported group treatments produced by the American Psychological Association's Task Force on Promotion and Dissemination of Psychological Procedures [43]. All of the studies reported statistically significant results; however, none had used the correct dfs or accounted for the within-group ICC. Baldwin et al. estimated statistical significance using the correct dfs and varying assumptions about the ICC [40]. After the correct dfs was used, only 50.8 to 68.2 percent of the previously significant findings remained significant, depending on assumptions made about the tests. In fact, if the ICC were a mere 0.05 , only 35.2 to 43.3 percent of the findings would have remained significant.

Only three of the RCTs listed in Tables $\mathbf{1}$ and $\mathbf{2}$ $[9,13,16]$ had corrected for the group clustering effect. Extrapolating from Baldwin et al.'s findings, this likely means that the current data on group therapy for PTSD overestimate the true effect of group therapy for PTSD [40]. In their meta-analysis, Sloan et al. reanalyzed data to correct for the group effect in studies that had not done so (see Sloan et al. for details on how the group clustering effect was corrected) [27].

\section{Missing Data}

Of the 17 studies included in Sloan et al.'s metaanalysis, 11 used an ITT analysis approach, although the majority used the last-endpoint-carried-forward (LOCF) method, which can result in substantial bias [27]. Although the conventional wisdom is that this method is conservative (and if anything, underestimates treatment effects), it can cause serious overestimation depending on the dropout rate and the pattern of symptom change in dropouts and completers. In its review of the evidence on PTSD treatment, the Institute of Medicine recommended that the LOCF method should not be used if the amount of dropout is over 10 percent, which is often the case in psychotherapy (and medication) studies [3]. Examination of completer data can be informative in terms of understanding what type of participants are likely to remain in treatment relative to those who are likely to drop out of treatment. However, ITT analysis with correct methods for handling missing data should be used to obtain an unbiased estimate of a treatment's benefit $[3,38]$.

\section{DISCUSSION}

It is important to carefully consider the conclusions that can be drawn from the meta-analytic findings of Sloan et al. [27]. First, group treatment is an effective approach for treating PTSD, although the obtained effect sizes suggest it may not be as effective as individual therapy. Second, none of the group treatments studied, which included CBT, interpersonal, and other approaches, demonstrated unique benefits beyond the general benefits of group therapy. The latter conclusion indicates a marked difference with the literature on individual treatment, in which CBT approaches have been shown to have robust unique effects in treating PTSD.

Although the meta-analytic findings for PTSD group treatments are not very encouraging, several issues should be considered. First, many of the studies had small sample sizes and, consequently, the total $N$ for the analyses was relatively small. In addition, the studies are heterogeneous in the terms of the type of target treatment, the trauma sample examined, and the outcome measure used. Moreover, studies that combined group and individual treatment were not included in the meta-analysis $[19,22]$. One possible direction is to focus future research efforts on the treatments that have the largest effects. In Sloan et al.'s meta-analysis [27], the studies that had within-group effect sizes larger than 1.0 were Beck et al. $(d=1.26)$ [13], Krupnick et al. $(d=1.13)$ [10], and Hien et al. $(d=1.01)$ [25]. Of these, only Krupnick et al. had a between-group effect size $(d=0.91)$ defined as large according to Cohen [44], but it was based on a wait list comparison condition and was substantially smaller than effect sizes reported for evidence-based individual treatments for PTSD when wait list comparison conditions are used [37]. Therefore, we believe it is to also important to focus future research on additional approaches.

\section{WHAT WE DO NOT KNOW ABOUT GROUP TREATMENT FOR PTSD}

Caution is needed when interpreting the research literature on group treatment for PTSD. For instance, a perceived advantage of group treatment is the increase in social support or social contact with other group members. However, very few studies have examined social functioning or social support as an outcome measure. Further, the studies that have examined social functioning 
have obtained mixed results. Classen et al. did not find that participants in the group treatment condition (both trauma-focused and present-centered treatments) differed in social functioning following treatment from participants in the wait list condition [9]. In contrast, Krupnick et al. found that participants in interpersonal therapy demonstrated improvements in social functioning relative to participants assigned to a wait list condition [10]. Similarly, Beidel et al. found that participants who completed trauma management therapy had improvements in social functioning at posttreatment relative to participants who completed an exposure-only treatment [22]. Importantly, the two studies that reported improvements in social functioning included social skills training as part of the treatment.

Another primary perceived advantage of group treatment is cost-effectiveness, yet no studies have examined the cost-effectiveness of group treatment for PTSD. Consequently, it is unknown whether group treatment is actually more cost-effective than individual treatment.

In addition, we do not know whether group treatment for PTSD is as effective as individual treatment for PTSD. Beck et al. [13] examined the efficacy of group treatment for motor-vehicle related PTSD that was based on an individually-delivered treatment developed by Blanchard et al. [45]. Although Beck et al. found that the group treatment was effective compared with a wait list condition, they did not directly compare the treatment to the individual format [13]. As previously stated, to date, no study has directly compared group treatment for PTSD with individual treatment to PTSD, although this research is ongoing.

A challenging aspect of treating PTSD is the high comorbidity rate of the disorder. Common comorbid conditions include substance use disorder, traumatic brain injury, and depression. We have limited information on how these comorbid conditions might affect group treatment outcomes, other than the general considerations that significant cognitive impairment and substance dependence might negatively affect a patient's ability to gain benefit from the group process. There are some treatments that target comorbid substance use disorder and PTSD, such as Najavits et al. [46]. However, the available evidence indicates that "Seeking Safety" group treatment is as effective as psychoeducational group treatments [25]. A group treatment that targets comorbid depression among veterans with PTSD has also been studied [28]. This study found a moderate effect size for improvement in depression symptoms at posttreatment relative to a psychoeducational comparison condition. Despite these encouraging results, this effect was no longer significant at a 3-month follow-up assessment, and no significant group difference was observed for PTSD symptom severity. Taken together, the treatments that have been developed to address comorbid conditions in PTSD have demonstrated limited efficacy.

We also do not know whether group treatment increases treatment retention or whether group treatment for PTSD results in increased treatment engagement, such as increased medication compliance. Patients might be more likely to remain in group treatment because of a sense of commitment to other group members. Generally, treatment dropout rates for group treatment for PTSD reported in the literature range considerably, but the average dropout rate is consistent with that of individual treatment for PTSD (e.g., 26\% as reported by Sloan et al. [27]).

The information we have regarding treatment satisfaction is very positive. Several of the RCTs included treatment satisfaction measures [11,13,28,31,33,36] and found that participants reported a positive experience with group treatment and a perceived benefit from the treatment. The positive treatment satisfaction ratings raise the possibility that patients are benefiting in ways that may not be captured by the outcome measures included in existing clinical trials. It is important to further examine how patients are benefiting from group treatment and to then include outcome measures that reflect the domain of benefit that patients report. For example, patients may perceive benefits in terms of increased social contact and normalization of PTSD symptoms.

\section{CONCLUSIONS AND RECOMMENDATIONS}

PTSD is an important public health issue given its prevalence in the general population, as well as the high incidence in the veteran population [47]. Because group treatment for PTSD is used frequently, it is surprising that greater attention has not been given to identifying evidence-based group treatment for this disorder. Part of the reason that we may not have advanced in this area is that group clinical trials are complex and can be expensive to conduct, given that the sample sizes needed are much 
larger than those needed for studies of individual treatment $[34,38]$.

More than 10 years ago, Foy et al. noted that the scientific literature on group treatment for PTSD had substantially lagged behind the literature on individual treatment [6]. This is still the case, although research on group treatment has increased substantially, with 14 RCTs published since Foy et al.'s article. Based on the existing research, group treatment for PTSD appears to be an effective approach, although meta-analytic findings suggest that it is not as effective as individual therapy [27]. Several treatments appear promising $[10,13,25]$, but focusing only on these treatments would be premature. Future research should consider additional approaches.

Given the limitations of the research literature, there is little evidence to guide clinicians on what group treatments for PTSD should be used and when they should be applied. Nonetheless, general guidelines exist for evaluating whether or not a particular patient is appropriate for group treatment [6,48]. First, it is important to consider the composition of the group members when deciding whether or not a specific patient might be suitable for a group. For example, it is advisable to avoid having a single member of the group that differs in some important way from other group members, such as sex or type of trauma. Second, patients who are severely depressed or psychotic, have severe cognitive impairment, or are currently in a chaotic or unstable (e.g., homeless) situation would not likely benefit from group treatment and may be disruptive to the group process. Third, some patients do not feel comfortable in a group setting, and this discomfort may inhibit them from actively engaging in group treatment. Fourth, clinicians should consider current substance use and personality traits of patients. If patients are actively abusing substances or if they have paranoid or sociopathic personality traits, they are unlikely to benefit from the group treatment setting and may be disruptive to the group. Patients may also not be suitable for group treatment if they have restrictive schedules. In general, scheduling group treatment is more difficult than individual treatment because of the need to accommodate the schedules of all group members.

Having established that a patient is appropriate for a group treatment setting, the clinician must then consider the treatment goals for the patient and the patient's willingness to engage in group treatment. Anger might be the primary target of treatment for a patient; in this case, anger management group might be a good treatment choice. Another patient may need a comprehensive trauma-focused treatment but is unwilling to engage in a trauma-focused group treatment. In this case, individual treatment would be the best option for the patient.

Clinicians might also consider combining group treatment and individual treatment. For instance, Prolonged Exposure might be delivered individually while the patient also attends an anger management group [49]. Using both individual and group treatment approaches would allow the patient the opportunity to maximize treatment effects by addressing more than one treatment target area.

Overall, research on group treatment for PTSD has advanced over the past 20 years but not at the same rate as research on individual treatment. Additional work is needed to examine group treatment in combination with individual treatment, outcome measures other than PTSD (such as social functioning, treatment adherence, and engagement), and the cost-effectiveness of group treatment relative to individually delivered treatment.

\section{ACKNOWLEDGMENTS}

\section{Author Contributions:}

Study concept and design: P. P. Schnurr, D. M. Sloan. Acquisition of data: D. M. Sloan, M. J. Bovin.

Interpretation of data: D. M. Sloan, M. J. Bovin, P. P. Schnurr. Drafting of manuscript: D. M. Sloan, M. J. Bovin, P. P. Schnurr. Critical revision of manuscript for important intellectual content: D. M. Sloan, M. J. Bovin, P. P. Schnurr.

Financial Disclosures: The authors have declared that no competing interests exist.

Funding/Support: This article was based on work supported in part by a National Institute of Mental Health T32 grant (grant T32MH019836-14A2).

Additional Contributions: The authors thank Danny Kaloupek for his comments on a previous draft of this manuscript.

\section{REFERENCES}

1. Shapiro F. Eye movement desensitization and reprocessing: Basic principles, protocols, and procedures. New York (NY): Guilford Press; 1995.

2. Friedman MJ, Cohen JA, Foa EB, Keane TM. Integration and summary. In: Foa EB, Keane TM, Friedman MJ, Cohen JA, editors. Effective treatments for PTSD: Practice guidelines from the International Society for Traumatic Stress Studies. 2nd ed. New York (NY): Guilford Press; 2009. p. 617-42. 
3. Institute of Medicine. Treatment of posttraumatic stress disorder: An assessment of the evidence. Washington (DC): National Academies Press; 2007.

4. Management of Post-Traumatic Stress Working Group (Internet). VA/DOD clinical practice guideline for management of post-traumatic stress. Washington (DC): Department of Veterans Affairs, Department of Defense; 2010. Available from: http://www.healthguality.va.gov/ ptsd/ptsd full.pdf

5. Forbes D, Creamer M, Bisson JI, Cohen JA, Crow BE, Foa EB, Friedman MJ, Keane TM, Kudler HS, Ursano RJ. A guide to guidelines for the treatment of PTSD and related conditions. J Trauma Stress. 2010;23(5):537-52.

[PMID:20839310]

http://dx.doi.org/10.1002/jts.20565

6. Foy DW, Glynn SM, Schnurr PP, Jankowski MK, Wattenberg MS, Weiss DS, et al. Group therapy. In: Foa EB, Keane TM, Friedman, MJ, editors. Effective treatments for PTSD: Practice guidelines from the International Society for Traumatic Stress Studies. New York (NY): Guilford Press; 2000. p. 155-75.

7. Hunt MG, Rosenheck RA. Psychotherapy in mental health clinics of the Department of Veterans Affairs. J Clin Psychol. 2011;67(6):561-73. [PMID:21416465] http://dx.doi.org/10.1002/jclp.20788

8. Yalom ID. The theory and practice of group psychotherapy. 4th ed. New York (NY): Basic Books; 1995.

9. Classen CC, Palesh OG, Cavanaugh CE, Koopman C, Kaupp JW, Kraemer HC, Aggarwal R, Spiegel D. A comparison of trauma-focused and present-focused group therapy for survivors of childhood sexual abuse: A randomized controlled trial. Psychol Trauma. 2011;3(1):84-93.

http://dx.doi.org/10.1037/a0020096

10. Krupnick JL, Green BL, Stockton P, Miranda J, Krause E, Mete M. Group interpersonal psychotherapy for low-income women with posttraumatic stress disorder. Psychother Res. 2008;18(5):497-507. [PMID:18816001] http://dx.doi.org/10.1080/10503300802183678

11. Morland LA, Greene CJ, Rosen CS, Foy D, Reilly P, Shore J, He Q, Frueh BC. Telemedicine for anger management therapy in a rural population of combat veterans with posttraumatic stress disorder: A randomized noninferiority trial. J Clin Psychiatry. 2010;71(7):855-63.

[PMID:20122374] http://dx.doi.org/10.4088/JCP.09m05604blu

12. Hinton DE, Hofmann SG, Rivera E, Otto MW, Pollack MH. Culturally adapted CBT (CA-CBT) for Latino women with treatment-resistant PTSD: A pilot study comparing CA-CBT to applied muscle relaxation. Behav Res Ther. 2011;49(4):275-80. [PMID:21333272] http://dx.doi.org/10.1016/j.brat.2011.01.005
13. Beck JG, Coffey SF, Foy DW, Keane TM, Blanchard EB. Group cognitive behavior therapy for chronic posttraumatic stress disorder: An initial randomized pilot study. Behav Ther. 2009;40(1):82-92. [PMID:19187819] http://dx.doi.org/10.1016/j.beth.2008.01.003

14. Bradley RG, Follingstad DR. Group therapy for incarcerated women who experienced interpersonal violence: A pilot study. J Trauma Stress. 2003;16(4):337-40.

[PMID:12895016] http://dx.doi.org/10.1023/A:1024409817437

15. Ready DJ, Thomas KR, Worley V, Backscheider AG, Harvey LA, Baltzell D, Rothbaum BO. A field test of group based exposure therapy with 102 veterans with war-related posttraumatic stress disorder. J Trauma Stress. 2008;21(2): 150-57. [PMID:18404634]

http://dx.doi.org/10.1002/jts.20326

16. Schnurr PP, Friedman MJ, Foy DW, Shea MT, Hsieh FY, Lavori PW, Glynn SM, Wattenberg M, Bernardy NC. Randomized trial of trauma-focused group therapy for posttraumatic stress disorder: Results from a Department of Veterans Affairs cooperative study. Arch Gen Psychiatry. 2003;60(5):481-89. [PMID:12742869]

http://dx.doi.org/10.1001/archpsyc.60.5.481

17. Resick PA, Schnicke MK. Cognitive processing therapy for sexual assault victims. J Consult Clin Psychol. 1992;60(5): 748-56. [PMID:1401390] http://dx.doi.org/10.1037/0022-006X.60.5.748

18. Resick PA, Nishith P, Weaver TL, Astin MC, Feuer CA. A comparison of cognitive-processing therapy with prolonged exposure and a waiting condition for the treatment of chronic posttraumatic stress disorder in female rape victims. J Consult Clin Psychol. 2002;70(4):867-79. [PMID:12182270] http://dx.doi.org/10.1037/0022-006X.70.4.867

19. Chard KM. An evaluation of cognitive processing therapy for the treatment of posttraumatic stress disorder related to childhood sexual abuse. J Consult Clin Psychol. 2005;73(5): 965-71. [PMID:16287396] http://dx.doi.org/10.1037/0022-006X.73.5.965

20. Hebert M, Rosenheck R, Drebing C, Young AL, Armstrong M. Integrating peer initiatives in a large healthcare organization. Psychol Serv. 2008;5:216-27.

http://dx.doi.org/10.1037/1541-1559.5.3.216

21. Turner SM, Beidel DC, Frueh BC. Multicomponent behavioral treatment for chronic combat-related posttraumatic stress disorder: Trauma management therapy. Behav Modif. 2005;29(1):39-69. [PMID:15557478] http://dx.doi.org/10.1177/0145445504270872

22. Beidel DC, Frueh BC, Uhde TW, Wong N, Mentrikoski JM. Multicomponent behavioral treatment for chronic combat-related posttraumatic stress disorder: A randomized controlled trial. J Anxiety Disord. 2011;25(2):224-31. 


\section{[PMID:20951543]}

http://dx.doi.org/10.1016/j.janxdis.2010.09.006

23. Resick PA, Schnicke MK. Cognitive processing therapy for rape victims: A treatment manual. Newbury Park (CA): Sage Publications; 1993.

24. Zlotnick C, Shea TM, Rosen K, Simpson E, Mulrenin K, Begin A, Pearlstein T. An affect-management group for women with posttraumatic stress disorder and histories of childhood sexual abuse. J Trauma Stress. 1997;10(3):425-36. [PMID:9246650]

http://dx.doi.org/10.1002/jts.2490100308

25. Hien DA, Wells EA, Jiang H, Suarez-Morales L, Campbell AN, Cohen LR, Miele GM, Killeen T, Brigham GS, Zhang Y, Hansen C, Hodgkins C, Hatch-Maillette M, Brown C, Kulaga A, Kristman-Valente A, Chu M, Sage R, Robinson JA, Liu D, Nunes EV. Multisite randomized trial of behavioral interventions for women with co-occurring PTSD and substance use disorders. J Consult Clin Psychol. 2009; 77(4):607-19. [PMID:19634955]

http://dx.doi.org/10.1037/a0016227

26. Shea MT, McDevitt-Murphy M, Ready DJ, Schnurr PP. Group therapy. In: Foa EB, Keane TM, Friedman MJ, Cohen JA, editors. Effective treatments for PTSD: Practice guidelines from the International Society for Traumatic Stress Studies. 2nd ed. New York (NY): Guilford Press; 2009. p. 306-26.

27. Sloan DM, Feinstein BA, Gallagher MW, Beck JG, Keane TM. Efficacy of group treatment for posttraumatic stress disorder symptoms: A meta-analysis. Psychol Trauma. 2011 Nov 7. http://dx.doi.org/10.1037/a0026291

28. Dunn NJ, Rehm LP, Schillaci J, Souchek J, Mehta P, Ashton CM, Yanasak E, Hamilton JD. A randomized trial of self-management and psychoeducational group therapies for comorbid chronic posttraumatic stress disorder and depressive disorder. J Trauma Stress. 2007;20(3):221-37. [PMID:17598141] http://dx.doi.org/10.1002/jts.20214

29. Falsetti SA, Resnick HS, Davis JL. Multiple channel exposure therapy for women with PTSD and comorbid panic attacks. Cogn Behav Ther. 2008;37(2):117-30. [PMID:18470742] http://dx.doi.org/10.1080/16506070801969088

30. Harris JI, Erbes CR, Engdahl BE, Thuras P, Murray-Swank N, Grace D, Ogden RH, Winskowski AM, Bacon R, Malec C, Campion K, Le T. The effectiveness of a trauma focused spiritually integrated intervention for veterans exposed to trauma. J Clin Psychol. 2011;67(4):425-38.

[PMID:21294116]

http://dx.doi.org/10.1002/jclp.20777

31. Hollifield M, Sinclair-Lian N, Warner TD, Hammerschlag R. Acupuncture for posttraumatic stress disorder: A randomized controlled pilot trial. J Nerv Ment Dis. 2007;
195(6):504-13. [PMID:17568299]

http://dx.doi.org/10.1097/NMD.0b013e31803044f8

32. Krakow B, Hollifield M, Schrader R, Koss M, Tandberg D, Lauriello J, McBride L, Warner TD, Cheng D, Edmond T, Kellner R. A controlled study of imagery rehearsal for chronic nightmares in sexual assault survivors with PTSD: A preliminary report. J Trauma Stress. 2000;13(4):589609. [PMID:11109233] http://dx.doi.org/10.1023/A:1007854015481

33. Rogers S, Silver SM, Goss J, Obenchain J, Willis A, Whitney RL. A single session, group study of exposure and Eye Movement Desensitization and Reprocessing in treating posttraumatic stress disorder among Vietnam war veterans: Preliminary data. J Anxiety Disord. 1999;13(1-2):119-30. [PMID:10225504] http://dx.doi.org/10.1016/S0887-6185(98)00043-7

34. Schnurr PP, Friedman MJ, Lavori PW, Hsieh FY. Design of Department of Veterans Affairs Cooperative Study No. 420: Group treatment of posttraumatic stress disorder. Control Clin Trials. 2001;22(1):74-88. [PMID:11165426] http://dx.doi.org/10.1016/S0197-2456(00)00118-5

35. Sikkema KJ, Hansen NB, Kochman A, Tarakeshwar N, Neufeld S, Meade CS, Fox AM. Outcomes from a group intervention for coping with HIV/AIDS and childhood sexual abuse: Reductions in traumatic stress. AIDS Behav. 2007;11(1):49-60. [PMID:16858634] http://dx.doi.org/10.1007/s10461-006-9149-8

36. Zlotnick C, Johnson J, Najavits LM. Randomized controlled pilot study of cognitive-behavioral therapy in a sample of incarcerated women with substance use disorder and PTSD. Behav Ther. 2009;40(4):325-36. [PMID:19892078] http://dx.doi.org/10.1016/j.beth.2008.09.004

37. Cahill SP, Rothbaum BO, Resick PA, Folette VM. Cognitive-behavioral therapy for adults. In: Foa EB, Keane TM, Friedman MJ, Cohen JA, editors. Effective treatments for PTSD: Practice guidelines from the International Society for Traumatic Stress Studies. 2nd ed. New York (NY): Guilford Press; 2009. p. 139-222.

38. Schnurr PP. The rocks and hard places in psychotherapy outcome research. J Trauma Stress. 2007;20(5):779-92. [PMID:17955539] http://dx.doi.org/10.1002/jts.20292

39. Greene CJ, Morland LA, Durkalski VL, Frueh BC. Noninferiority and equivalence designs: Issues and implications for mental health research. J Trauma Stress. 2008;21(5): 433-39. [PMID:18956449]

http://dx.doi.org/10.1002/jts.20367

40. Baldwin SA, Murray DM, Shadish WR. Empirically supported treatments or type I errors? Problems with the analysis of data from group-administered treatments. J Consult Clin Psychol. 2005;73(5):924-35. [PMID:16287392] http://dx.doi.org/10.1037/0022-006X.73.5.924 
41. Burlingame GM, Kircher JC, Honts CR. Analysis of variance versus bootstrap procedures for analyzing dependent observations in small group research. Small Group Res. 1994;25:486-501. http://dx.doi.org/10.1177/1046496494254004

42. Creamer M, Morris P, Biddle D, Elliott P. Treatment outcome in Australian veterans with combat-related posttraumatic stress disorder: A cause for cautious optimism? J Trauma Stress. 1999;12(4):545-58. [PMID:10646175] http://dx.doi.org/10.1023/A:1024702931164

43. Sanderson WC, Woody S, editors. Manuals for empirically validated treatments [Internet]. Washington (DC): American Psychological Association; 1995 Jun. Available from: http://www.apa.org/divisions/div12/est/ MANUALSforevt.html

44. Cohen J. Statistical power analysis for the behavioral sciences. 2nd ed. Hillsdale (NJ): Erlbaum; 1988.

45. Blanchard EB, Hickling EJ, Devineni T, Veazey $\mathrm{CH}$, Galovski TE, Mundy E, Malta LS, Buckley TC. A controlled evaluation of cognitive behavioural therapy for posttraumatic stress in motor vehicle accident survivors. Behav Res Ther. 2003;41(1):79-96. [PMID:12488121] http://dx.doi.org/10.1016/S0005-7967(01)00131-0

46. Najavits LM, Weiss RD, Shaw SR, Muenz LR. "Seeking safety": Outcome of a new cognitive-behavioral psychotherapy for women with posttraumatic stress disorder and substance dependence. J Trauma Stress. 1998;11(3):437-56.

\section{[PMID:9690186]}

http://dx.doi.org/10.1023/A:1024496427434

47. Keane TM, Marx BP, Sloan DM. Posttraumatic stress disorder: Definition, prevalence and risk factors. In: Shiromani P, Keane TM, LeDoux J, editors. Posttraumatic stress disorder: Basic science and clinical practice. New York (NY): Humana Press; 2009. p. 1-22.

48. Yalom ID, Leszcz M. Theory and practice of group psychotherapy. 5th ed. New York (NY): Basic Books; 2005.

49. Foa EB, Rothbaum BO. Treating the trauma of rape: Cognitive-behavioral therapy for PTSD. New York (NY): Guilford Press; 1998.

Submitted for publication July 12, 2011. Accepted in revised form February 23, 2012.

This article and any supplementary material should be cited as follows:

Sloan DM, Bovin MJ, Schnurr PP. Review of group treatment for PTSD. J Rehabil Res Dev. 2012;49(5):689-702. http://dx.doi.org/10.1682/JRRD.2011.07.0123

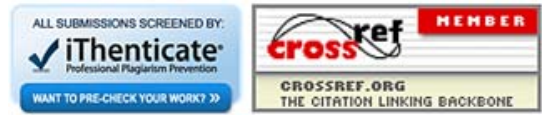


